\title{
Thickness-Dependent Thermal Behavior due to Molecular Tilt Coupling Strength in Free-Standing 70.7 Thin Films
}

\author{
Chih-Yu Chao \\ Tung-Cheng Pan \\ Ming-Tsong Hsu \\ Wen-Jiunn Hsieh
}

Department of Physics, National Taiwan University, Taipei, Taiwan, Republic of China

We report for the first time the thermal study near the Sm-C-Sm-I critical point in free-standing thin films of 70.7 compound. The profiles of the Sm-C-Sm-I heat-capacity anomalies are found to exhibit an unexpected crossover behavior with film thickness in reduced dimensions. The data from two-layer films are not consistent with the predictions of two-dimensional melting theory. Our results also provide evidence in support of the recent theory proposed by Defontaines and Prost. Moreover, we confirm the existence of a surface hexatic Sm-L phase in 70.7 films of less than eight layers whose phase sequence is however dramatically different from that observed in the thicker films.

Keywords: electron diffraction; high-resolution ac calorimetry; reduced dimensions; $\mathrm{Sm}-\mathrm{C}-\mathrm{Sm}-\mathrm{I}$ phase transition; smectic free-standing liquid-crystal thin films; tilt-induced bond-orientational order

Some smectic phases, known as hexatic, possess a long-range orientational order of the bonds (the lines connecting neighboring molecules), but only short-range positional order. The molecules can be either perpendicular to the layers (hexatic- $B$ ), or tilted with respect to the normal to the layers (smectic- $I$ and smectic- $F$ ) [1]. The vector characterizing the direction in which the molecules are tilted points towards the closest molecule in the smectic- $I$ (Sm-I) phase, and between the two closest molecules in the smectic- $F(\mathrm{Sm}-F)$ phase.

We are grateful to Profs. J. T. Ho and C. C. Huang for helpful discussions. This work was funded by the National Science Council, Taiwan, Republic of China.

Address correspondence to Chih-Yu Chao, Department of Physics, National Taiwan University, Taipei 10617, Taiwan. E-mail: cychao@ntu.edu.tw 
A new hexatic smectic- $L$ (Sm- $L$ ) phase, in which the tilt direction lies along an intermediate angle between $0^{\circ}$ and $30^{\circ}$ from a local bond, has also been theoretically predicted [2] and suggested in some experiments [3-5]. To date, this hexatic Sm-L phase was identified only in films of 5-(4'-hexyl, $3^{\prime}$-fluoro-p-terphenyl-4-oxy)-pentanoic acid ethyl ester (FTE1) by electron diffraction (ED) [6].

For the tilted hexatic phases, theory [7] predicts that the coupling between the bond-orientational (BO) order in the Sm-I and the molecular tilt in the smectic- $C(\mathrm{Sm}-C)$ phase has a profound effect on the nature of the tilted liquid-hexatic transition by inducing $\mathrm{BO}$ order in the Sm- $C$ phase and formally destroying the Sm-C-Sm- $I$ phase boundary. This prediction has been supported by X-ray [8] and heat-capacity $(\mathrm{Cp})$ [9] studies in 4-(2-methylbutyl)-phenyl-4'-(octyloxy)-(1,1')-biphenyl-4carboxylate (8OSI), which exhibits a continuous Sm- $C$-Sm- $I$ evolution. In this Cp experiment, Yao and Garland et al. [9] have reported that the occurrence of intrinsically rounded and asymmetric Sm-C-Sm-I $\mathrm{Cp}$ peak found in 8OSI is due to the existence of a finite coupling between the BO order and molecular tilt ordering, and predicted a sharp and symmetric $\mathrm{Cp}$ peak to appear in the absence of a coupling field to the BO order. More recently, theory by Defontaines and Prost (DP) [10] predicts that the Sm-C-Sm- $I$ transition is similar to that of a liquid-gas transition, with a first-order line ending in an isolated critical point. There can be either a first-order transition between $\mathrm{Sm}-C$ and $\mathrm{Sm}-\mathrm{I}$ or no thermodynamic phase transition, but a supercritical evolution of both $\mathrm{BO}$ order and tilt in a single phase. The existence of the critical Sm-C-Sm-I point has been established by calorimetry experiments in a racemic mixture of 8OSI and its analog [11] and by X-ray experiments, carried out on binary mixtures of terepthalbis-decylaniline (TB10A) and 4-n-decyloxy biphenyl 4-(2'-methylbutyl) benzoate (C10) [12]. The X-ray result has shown that the critical exponents near the Sm-C-Sm-I critical point are comparable to the values for new universality class predicted by DP. In the other words, the light scattering study [13] have shown that the coupling between BO order and tilt is smaller by a factor of $10^{2}$ in 8SI than that in 8OSI, which supports that the observed Cp peak in pure 8SI [11] is quite sharp and symmetric. However, the thermal critical exponent obtained in $75 \%$ $8 \mathrm{OSI}$ mixture in the path of approach to the Sm-C-Sm-I critical point is found to be $1.06 \pm 0.08$ [11], which corresponds to a mean-field value rather than the value associated with the DP's new universality class. The first calorimetric study to date of the $\mathrm{Sm}-\mathrm{C}-\mathrm{Sm}-\mathrm{I}$ in thin free-standing films has revealed that the surface $\mathrm{Sm}-\mathrm{C}-\mathrm{Sm}-\mathrm{I} \mathrm{Cp}$ peak is seriously rounded although it is somewhat symmetric in thin films of $p$-decyloxybenzylidene- $p$-amino-2-methylbutylcinnamate 
(DOBAMBC), and in 4- and 12-layer films of 8OSI [14]. Such a rounding in the $\mathrm{Cp}$ anomaly not only gives fairly poor fitting to a power-law expression but also yields much difficulty to determine whether the $\mathrm{Sm}-\mathrm{C}-\mathrm{Sm}-\mathrm{I} \mathrm{Cp}$ profile is symmetric or asymmetric. However, there have been relatively few studies in thin films after that in the past.

The compound of 4- $n$-heptyloxybenzylidene-4- $n$-heptylaniline (70.7), which does not possess any hexatic phase in the bulk, exhibits a rich array of tilted hexatic behavior in free-standing films [15]. Films of six layers or less in the Sm- $C$ phase undergo transition upon further cooling to films with with Sm- $I$ surfaces and a Sm-C interior $[16,17]$. There was no evidence of induced BO order found in the $\mathrm{Sm}-C$ phase by the molecular tilt. However, we recently showed that that freestanding 70.7 films of 8 to 25 molecular layers possess a distinct tilted smectic- $C^{\prime}\left(\mathrm{Sm}-C^{\prime}\right)$ phase sandwiched between a Sm- $I$ surface layer and an orthogonal liquid interior [18]. This $\mathrm{Sm}-\mathrm{C}^{\prime}$ phase in 70.7 is a novel tilted liquid with hexaticlike positional correlations but no long-range $\mathrm{BO}$ order. Its behavior appears to be intermediate between that in 8OSI, where strong coupling between the tilt and BO orders results in the Sm-C phase being hexatic like [8], and that in FTE1, in which the orientations of the tilt and hexatic orders can be uncoupled $[6,8]$. Thus, thin free-standing films of such compound 70.7 represent a unique system with which to study the nature of the Sm-C-Sm-I critical point and the behavior of the Sm-C-Sm-I transition as a function of film thickness (reduced dimensionality). We have conducted high-resolution $\mathrm{Cp}$ measurements near the Sm-C-Sm- $I$ phase transition of thin free-standing 70.7 films and structural measurements using ED to identify the phases of these films. The profiles of the $\mathrm{Sm}-C-\mathrm{Sm}-I \mathrm{Cp}$ anomalies exhibit an interesting crossover behavior in reduced dimensions. The $\mathrm{Cp}$ peaks are found to be asymmetric in the thicker films, transforming to symmetric in films less than eight molecular layers. The sharp and slightly asymmetric $\mathrm{Cp}$ peak observed in the 8-layer film near the Sm-C-Sm-I critical point can be well fitted by a simple power-law expression with thermal critical exponent about 0.50 , which is different from the value obtained in the previous thermal experiments of the near-critical 8OSI mixtures [11] but in turn is in agreement with the theoretical prediction by DP [10]. Furthermore, our ED has shown the existence of a surface Sm- $L$ phase involving in the surface-freezing transitions in 70.7 films less than eight layers, that is however distinctly different from the phase-transitional sequence observed in thicker films.

Our experimental techniques for making Cp [19] and ED [20] measurements have been described elsewhere. The free-standing 70.7 films were drawn at about $80^{\circ} \mathrm{C}$ over a hole, with a diameter of 
$8 \mathrm{~mm}$ for $\mathrm{Cp}$ measurements and $1 \mathrm{~mm}$ for ED measurements. The electron-beam diameter used in this study was about $10 \mu \mathrm{m}$. The exact number of smectic layers $N$ in our films was determined by optical reflectivity [21]. We have studied in detail the structures of free-standing 70.7 films between 2 and 10 layers. For $N \geq 8$, the observed phases and transition sequence are still consistent to those of our previous ED study in 70.7 thicker films [18]. However, in films of thickness between 3 and 7 layers, our ED experiments reveal that the interior smectic- $A$ (Sm-A) liquid ring disappears and the typical variety of diffraction patterns observed on cooling from the $\mathrm{Sm}-C$ phase is illustrated in Figure 1 for a six-layer film. The diffraction pattern above $77.8^{\circ} \mathrm{C}$,

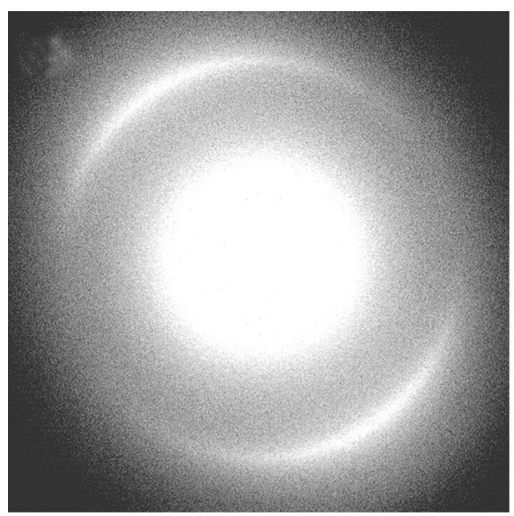

(a)

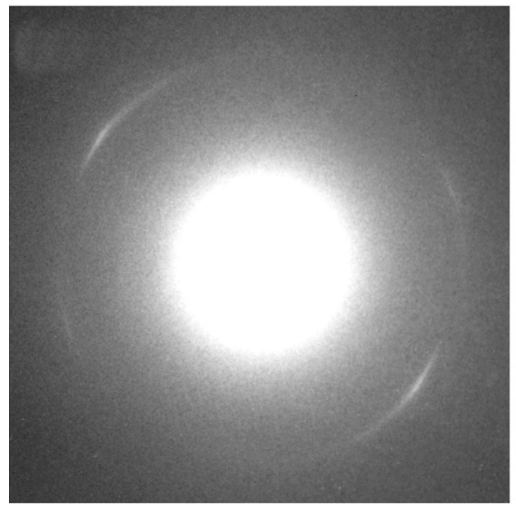

(c)

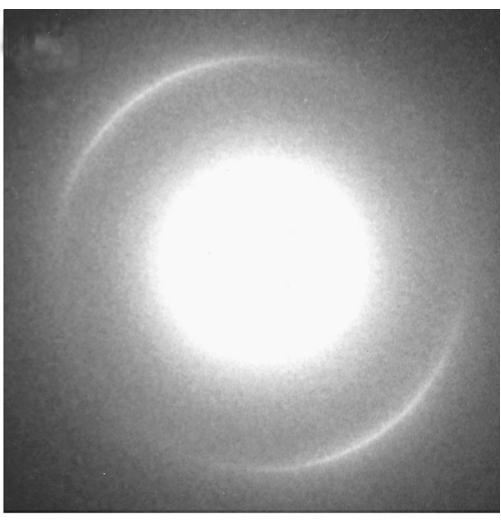

(b)

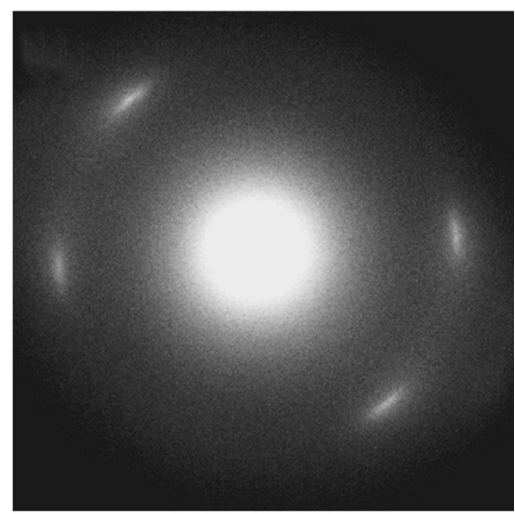

(d)

FIGURE 1 Electron-diffraction pattern from a six-layer film of 70.7 at (a) $78.5^{\circ} \mathrm{C}$, (b) $77.0^{\circ} \mathrm{C}$, (c) $74.0^{\circ} \mathrm{C}$, and (d) $64.5^{\circ} \mathrm{C}$. 
shown in Figure 1(a), consists of a diffuse ring acquiring an additional twofold intensity modulation characteristic of oriented Sm- $C$ ordering in the probed region. Upon further cooling, the diffraction pattern shown in Figure $1(\mathrm{~b})$ was observed between $77.8^{\circ} \mathrm{C}$ and $76^{\circ} \mathrm{C}$. The transition at about $77.8^{\circ} \mathrm{C}$ is characterized by an enhancement of the in-plane positional order on the surfaces while the interior remains in the $\mathrm{Sm}-\mathrm{C}$ phase. The radial scan of the intensity taken at $77^{\circ} \mathrm{C}$, shown in Figure 2, reveals the coexistence of a broad Lorentzian function with a positional correlation length of $10.2 \AA$, which is consistent with a liquidlike Sm- $C$ interior, and a narrow square-root Lorentzian function with a positional correlation length of $56.5 \AA$, which is typical of that found in a hexatic phase [22]. The presence of two strong, sharper arcs superimposed on the diffuse $\mathrm{Sm}-C$ ring suggests that the film surfaces are in the Sm-I phase. We note that the angular width of the sharper arcs in Figure 1(b) is somewhat broader than expected for a single-domain hexatic. Optically, we found that the film displays 3 - $\mu \mathrm{m}$-wide bands in which the director splays continuously, separated by disclination lines. The Sm-I arcs are most likely broadened by the finite range of bond orientations within the $10-\mu \mathrm{m}$ electron beam.

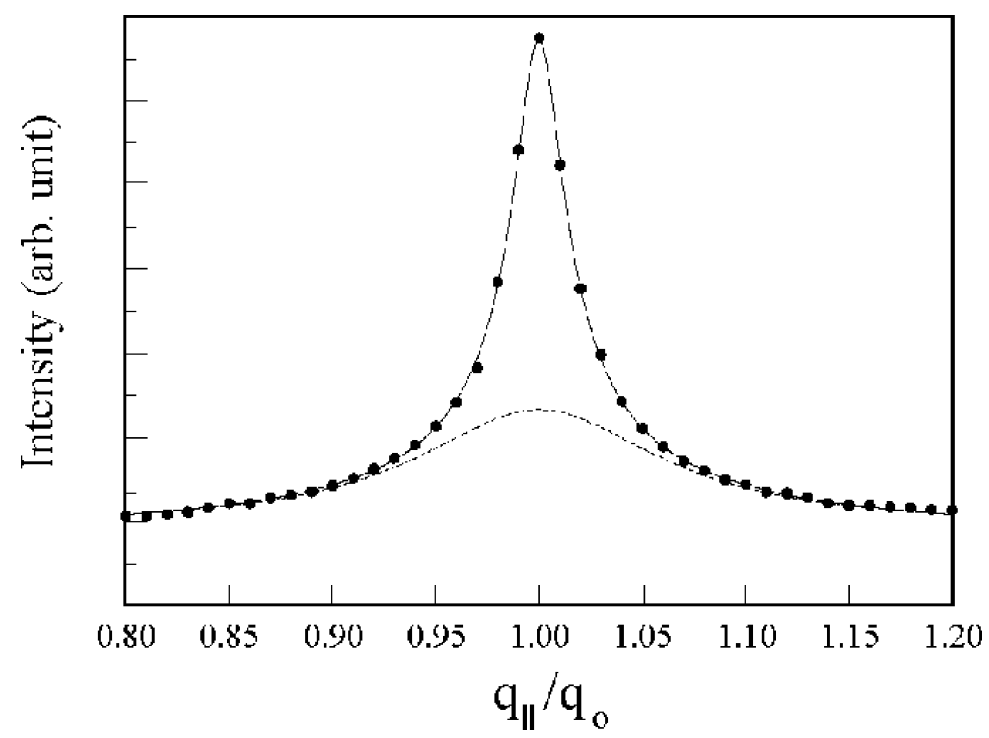

FIGURE 2 Diffraction intensity as a function of the in-plane radial wave vector $\mathrm{q}_{\|}$for a six-layer 70.7 film at $77.0^{\circ} \mathrm{C}$, normalized by the wave vector $\mathrm{q}_{\mathrm{o}}$ corresponding to the average nearest-neighbor spacing. The solid line is the fit to a sum of a broad Lorentzian function (dashed curve) and a narrow square-root Lorentzian function. 


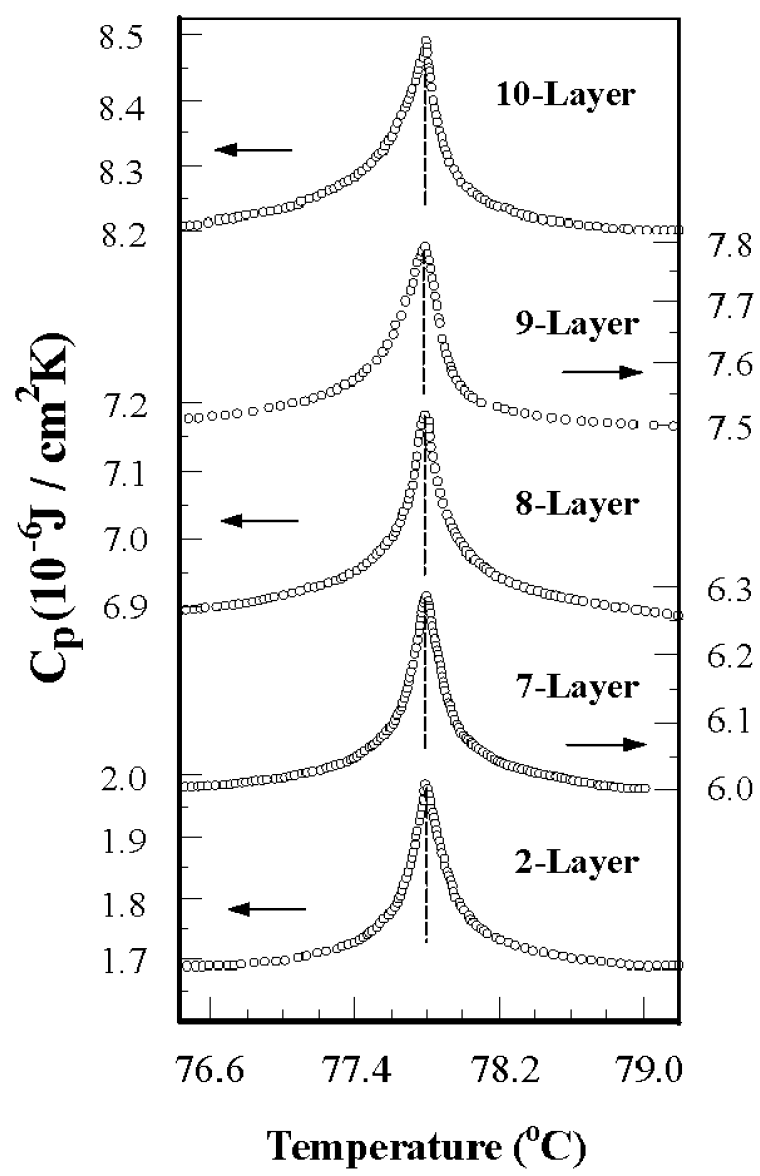

FIGURE 3 Heat-capacity data from 2- and 7-layer 70.7 films near the surface Sm- $C-\mathrm{Sm}-I$ transition and from 8-, 9-, and 10-layer films near the Sm-C-Sm$I$ (surface)/Sm- $C^{\prime}$ (interior) transition. For visual clarity, the heat-capacity data of 8-, 9-, and 10-layer films have been reduced by a factor of 3,3 , and 4 , respectively and the 2 -layer data set has been shifted by $-0.9^{\circ} \mathrm{C}$.

We also measured the Cp of these films near this surface $\mathrm{Sm}-\mathrm{C}-\mathrm{Sm}$ $I$ transition on successive cooling and heating runs. Figure 3 displays the temperature dependence of the $\mathrm{Cp}$ for 70.7 films of different layer thickness upon cooling. The observed $\mathrm{Cp}$ anomaly for $N<8$ in Figure 3 relates to the surface $\mathrm{Sm}-\mathrm{C}-\mathrm{Sm}-\mathrm{I}$ transition, whereas the $\mathrm{Cp}$ peak for $N \geq 8$ corresponds to a transition where the surface layers undergo the Sm-C-Sm- $I$ transition and the several adjacent-to-surface Sm-C layers being immediately induced some hexatic like order from the 
$\mathrm{Sm}-I$ surface transform into the $\mathrm{Sm}-C^{\prime}$ phase. The transition temperature is not changed as the films become thinner except for a slightly increase in the two-layer film. Unlike the previous thermal study of the Sm-C-Sm- $I$ transition in thin free-standing films [14], in which a noticeable rounding of the $\mathrm{Cp}$ anomaly was found in the surface transition, the surface anomaly in 70.7 is found to remain reasonably sharp in films down to two layers. The profiles of the Sm-C-Sm-I Cp anomalies exhibit an interesting crossover behavior in these thin films. In Figure 3, we find that the $\mathrm{Cp}$ peak is asymmetric for $N>8$, but becomes quite symmetric for $N \leq 8$. The Cp profile of the thinner films $(N \leq 8)$ appears to be a little narrower compared to that of the thicker films $(N>8)$. The crossover occurs at the 8-layer film whose detailed and enlarged $\mathrm{Cp}$ data are shown in Figure 4, in which a slightly asymmetric $\mathrm{Cp}$ profile is found. Interestingly, our $\mathrm{Cp}$ data in 70.7 films of different layer thickness are quite similar to those found in the previous bulk experiments in 8OSI and its racemic mixtures of different concentrations [11]. It should be noted that the Cp profile for $N>8$ is still reasonably sharp, not like the rounded $\mathrm{Cp}$ anomaly observed in the pure 8OSI bulk [11]. The reason may be due to the reason that the coupling between the $\mathrm{BO}$ order and molecular tilt in 70.7 films is smaller than that in 8OSI. However, we believe that

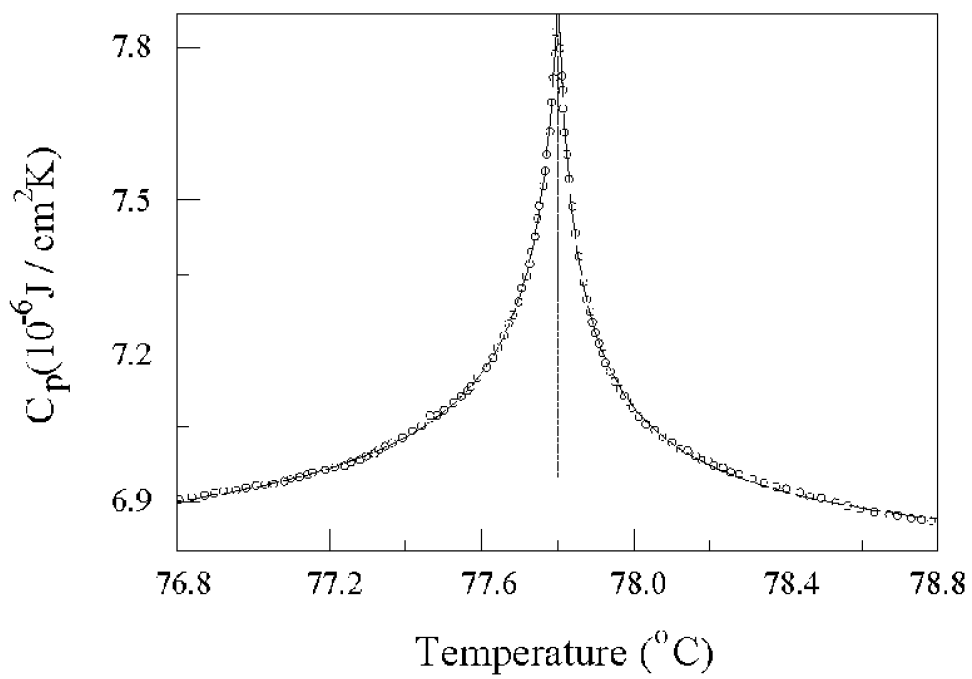

FIGURE 4 The fittings of the heat-capacity anomaly of the 8-layer 70.7 film to a simple power law are shown as solid lines and yield critical exponent $\alpha=0.51 \pm 0.03$. 
the asymmetry in $\mathrm{Cp}$ anomaly in 70.7 films for $N \geq 8$ could be still due to the formation of coupling between the $\mathrm{BO}$ and tilt orders, because there is possibility that the interior tilt-induced $\mathrm{Sm}-\mathrm{C}^{\prime}$ phase is the result of powder diffraction from a multidomain $\mathrm{Sm}-I$ spatially averaged by the electron beam of diameter $10 \mu \mathrm{m}$. Furthermore, the fact that the bulk $\mathrm{Cp}$ behaviors associated with the Sm-C-Sm-I transition $[9,11]$ can be similarly found in thin free-standing films may be due to the presence of more tilted surface layers provides stronger surface interactions to suppress the finite-size fluctuations and stabilize the thin films [23]. However, the reason for the disappearance of the coupling between the BO order and the tilt in $\mathrm{Sm}-\mathrm{C}$ phase for $N<8$ could be due to the expected increased fluctuations by the reduced dimensionality in thinner films. The fact that the $\mathrm{Cp}$ peak for $N<8$ expected to be very sharp based on the observations of the previous experiments [11] appears to be only reasonably sharp may be due to the influence of the finite-size rounding effect. The unique crossover phenomenon in the $\mathrm{Cp}$ profiles in thin free-standing 70.7 films illustrates the competition among surface interactions, low-dimensional fluctuations, and the coupling strength between BO and tilt orders.

To examine whether the $\mathrm{Cp}$ anomaly may be affected by the weakly first-order nature of the transition due to weaker coupling strength between the $\mathrm{BO}$ order and molecular tilt in 70.7 films, we have conducted detailed thermal hysteresis studies. From repeated runs using different films, we obtained the same Cp profiles on cooling and heating runs of about $3-5 \mathrm{mK} / \mathrm{min}$. Based on an upper-limit estimation, we found thermal hysteresis of 100 and $70 \mathrm{mK}$ for the transitions associated with the thinner $(N<8)$ and thicker films $(N \geq 8)$, respectively. Hence both of the Sm- $C-\mathrm{Sm}-I(N<8)$ and $\mathrm{Sm}-C-\mathrm{Sm}-I$ (surface)/Sm$C^{\prime}$ (interior) $(N \geq 8)$ transitions are at most weakly first order. Thus the observed change in $\mathrm{Cp}$ profile from asymmetric to symmetric one with decreasing film thickness appears to be an intrinsic phenomenon unrelated to the slightly first-order nature of the transition. Moreover, the width of the two-phase coexistence region for $N<8$ is estimated to be no larger than $55 \mathrm{mK}$ by taking closer examinations on the vicinity of the $\mathrm{Cp}$ peaks. The $\mathrm{Cp}$ data of the 8-layer film, shown in Figure 4, indicate a coexistence region smaller than $25 \mathrm{mK}$. For those 9- and 10-layer films, we found that there is no indication of two-phase coexistence. In these films, the reasonably sharp $\mathrm{Cp}$ profiles are smoothly rounded near the peak vicinity of $20 \mathrm{mK}$ or less. To understand the behavior of the Sm-C-Sm-I critical point, we have fitted $\mathrm{Cp}$ data of the critical 8-layer film thickness using a simple powerlaw expression and yielded a very good fit with the critical exponent 
$\alpha=0.51 \pm 0.03$, as shown in Figure 4. This result here represents the first thermal experiment that the value of critical exponent $\alpha$ in the vicinity of the Sm-C-Sm- $I$ critical point is close to 0.5 , in good agreement with the value for new universality class predicted by DP. We also found that the measured two-layer film anomaly is much larger than expected based on the present theory of two-dimensional (2D) melting [24], suggesting that the creation of BO order cannot be solely responsible for such a large anomaly and that some other type of molecular order must also be created through the $\mathrm{Sm}-\mathrm{C}-$ Sm- $I$ transition.

Upon further cooling to about $76^{\circ} \mathrm{C}$, the film undergoes another surface transition, giving a diffraction pattern shown in Figure 1(c), in which two pairs of sharper arcs of different intensity are superimposed on the diffuse Sm- $C$ modulated ring. A $\chi$ scan around the diffraction circle, as shown in Figure 5(a), reveals two pairs of uneven hexatic $\operatorname{arcs} 60^{\circ}$ apart and a broad, twofold Sm-C background. It has been suggested [25] and confirmed by numerical simulation [26] that the in-plane diffraction pattern of the $\mathrm{Sm}-L$ phase is characterized by three pairs of arcs of different intensity. Thus Figure 1(c) signifies the existence of $\mathrm{Sm}-L$ surface layers on top of the $\mathrm{Sm}-C$ interior. Optically, previous study [4] has shown that uniform stripes coexisting with $\mathrm{Sm}-C$-like director fluctuations in the background were observed in 70.7 films, that is in roughly the same temperature range in which the diffraction pattern in Figure 1(c) was observed. Hence our ED structural data in 70.7 films once again support the earlier suggestion that the stripes are due to the existence of a surface $\mathrm{Sm}-L$ phase $[4,6]$. However, our ED data are not in agreement with those in earlier X-ray experiments $[16,17]$ in that the 70.7 films of six layers or less have not revealed any evidence for the existence of the $\mathrm{Sm}-L$ phase. The discrepancy is probably due to the reason that the original X-ray phase identification was based primarily on radial scans, thus there is some uncertainty that the surface phase is really $\mathrm{Sm}-I$ [4]. The $\chi$-scan intensity after subtraction of the interior $\mathrm{Sm}-C$ contribution is shown as a function of temperature in Figure 5(b). The gradual shift in the intensity ratio of the two adjacent arcs on cooling suggests a continuous change of the tilt direction of the Sm- $L$ surfaces from Sm- $I$-like to $\mathrm{Sm}-F$-like, as expected theoretically [2]. It should be mentioned that no $\mathrm{Sm}-L$ phase was observed in the two-layer film in our ED experiments. This observation is consistent with that in FTE1 where no stripe phase was observed in two-layer films [27]. Finally, at around $66^{\circ} \mathrm{C}$, the entire film transforms into the $\mathrm{Sm}-F$ phase, characterized by a diffraction pattern with two pairs of arcs of equal intensity, as shown in Figure 1(d). Upon further cooling, we found that no 


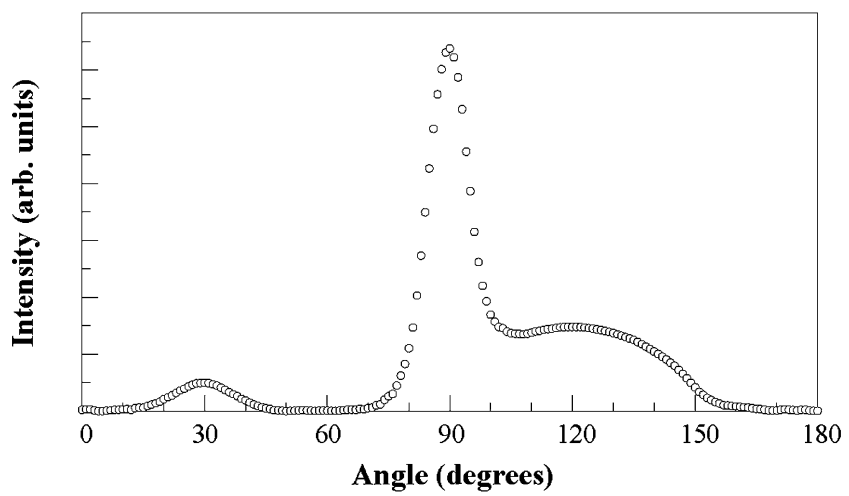

(a)

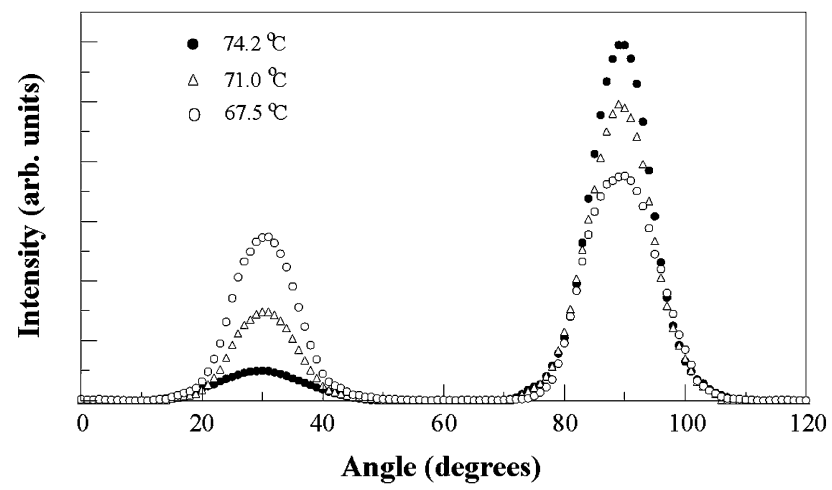

(b)

FIGURE 5 (a) Angular $\chi$ scan of diffraction intensity for a six-layer film at $74.0^{\circ}$ C. (b) Diffraction intensity along a $\chi$ scan for a six-layer film at various temperatures after subtraction of the background $\mathrm{Sm}-C$ contribution.

crystallization transition into the crystal- $B($ Cry- $B)$ phase at a lower temperature was observed in films of $N<8$, whose phase sequence is quite different from that in films of the same compound of $N \geq 8$ in which multiple-step freezing transitions lead to the transformation of these films to the Cry- $B$ phase $[5,18,28]$. We believe the suppression of the Cry- $B$ phase in thinner films $(N<8)$ is due to the possibility that the Cry- $B$ phase in 70.7 is intrinsically unstable with respect to tilted hexatic phase and that a relatively small decrease in the dimensionality of the films is sufficient to drive a transition from Cry- $B$ to tilted hexatic phases [23]. Nevertheless, the fact that our Cp data reveal the increased fluctuations by the reduced dimensionality for 
films of $N<8$ could also explain the suppression of Cry- $B$ phase in the thinner 70.7 films $(N<8)$ and the reason for the unusual occurrence of dramatically different phase sequences observed in the thinner $(N<8)$ and thicker $(N \geq 8) 70.7$ films. Furthermore, the reason for the unusual occurrence of the surface Sm- $L$ phase only in the thinner films $(2<N<8)$ can also be explained by the absence of coupling between the surface $\mathrm{BO}$ and interior tilt orders in these films, resulting in a fairly weak interaction between the two surfaces, and thus the molecular director is not constrained to be uniform through the film [4].

We report for the first time the detailed thermal study near the Sm$C-\mathrm{Sm}-I$ critical point in free-standing thin films of 70.7 compound, and find the profiles of the Sm-C-Sm-I Cp anomalies exhibit an unexpected crossover behavior with film thickness in reduced dimensions. The sharp and slightly asymmetric Cp peak of the 8-layer film near the $\mathrm{Sm}-\mathrm{C}-\mathrm{Sm}-\mathrm{I}$ critical point can be well characterized by a simple power-law expression. The obtained thermal critical exponent is in good agreement with the theoretical prediction by DP [10]. Our data also represent another structure evidence for the existence of the surface $\mathrm{Sm}-L$ phase in the thermotropic liquid-crystal system after the FTE1 and support the theoretical prediction [2] that, under some circumstances, a 2D tilted smectic liquid-crystal film can undergo a two-step transitions from the Sm- $I$ to $\mathrm{Sm}-F$ phases via an intermediate hexatic Sm- $L$ phase.

\section{REFERENCES}

[1] Chaikin, P. M. \& Lubensky, T. C. (1995). Principles of Condensed Matter Physics, Cambridge University Press: Cambridge, UK.

[2] Selinger, J. V. \& Nelson, D. R. (1988). Phys. Rev. Lett., 61, 416.

[3] Sirota, E. B., Smith, G. S., Safinya, C. R., Plano, R. J., \& Clark, N. A. (1988). Science, 242, 1406.

[4] Maclennan, J. E. \& Seul, M. (1992). Phys. Rev. Lett., 69, 2082; Maclennan, J. E., Sohling, U., Clark, N. A., \& Seul, M. (1994). Phys. Rev. E, 49, 3207.

[5] Chao, C. Y., Pan, T. C., \& Ho, J. T. (2003). Phys. Rev. E, 67, 040702(R).

[6] Chao, C. Y., Hui, S. W., Maclennan, J. E., Chou, C. F., \& Ho, J. T. (1997). Phys. Rev. Lett., 78, 2581.

[7] Nelson, D. R. \& Halperin, B. I. (1980). Phys. Rev. B, 21, 5312.

[8] Brock, J. D., Aharony, A., Birgeneau, R. J., Evans-Lutterodt, K. W., Litster, J. D., Horn, P. M., Stephenson, G. B., \& Tajbakhsh, A. R. (1986). Phys. Rev. Lett., 57, 98.

[9] Garland, C. W., Litster, J. D., \& Stine, K. J. (1989). Mol. Cryst. Liq. Cryst., 170, 71.

[10] Defontaines, A. D. \& Prost, J. (1993). Phys. Rev. E, 47, 1184.

[11] Yao, H., Chan, T., \& Garland, C. W. (1995). Phys. Rev. E, 51, 4585.

[12] Krishna, S., Shankar Rao, D. S., Chandrasekhar, S., Neubert, M. E., \& Goodby, J. W. (1995). Phys. Rev. Lett., 74, 270.

[13] Sprunt, S., Spector, M., \& Litster, J. D. (1992). Phys. Rev. A, 45, 7355.

[14] Stoebe, T. \& Huang, C. C. (1994). Phys. Rev. E, 50, R32. 
[15] Sirota, E. B., Pershan, P. S., Sorensen, L. B., \& Collett, J. (1987). Phys. Rev. A, $36,2890$.

[16] Sirota, E. B., Pershan, P. S., Amador, S., \& Sorensen, L. B. (1987). Phys. Rev. A, $35,2283$.

[17] Amador, S., Pershan, P. S., Stragier, H., Swanson, B. D., Tweet, D. J., Sorensen, L. B., Sirota, E. B., Ice, G.. E., \& Habenschuss, A. (1989). Phys. Rev. A, 39, 2703.

[18] Chao, C. Y., Hui, S. W., \& Ho, J. T. (1997). Phys. Rev. Lett., 78, 4962.

[19] Geer, R., Stoebe, T., Pitchford, T., \& Huang, C. C. (1991). Rev. Sci. Instrum., 62, 415.

[20] Cheng, M., Ho, J. T., Hui, S. W., \& Pindak, R. (1987). Phys. Rev. Lett., 59, 1112.

[21] Young, C. Y., Pindak, R., Clark, N. A., \& Meyer, R. B. (1978). Phys. Rev. Lett., 40, 773.

[22] Davey, S. C., Budai, J., Goodby, J. W., Pindak, R., \& Moncton, D. E. (1984). Phys. Rev. Lett., 53, 2129; Aeppli, G. \& Bruinsma, R. (1984). Phys. Rev. Lett., 53, 2133.

[23] de Jeu, W. H., Ostrovskii, B. I., \& Shalaginov, A. N. (2003). Rev. Mod. Phys., 75, 181.

[24] Kosterlitz, J. M. \& Thouless, D. J. (1973). J. Phys. C, 6, 1181; Nelson, D. R. \& Halperin, B. I. (1979). Phys. Rev. B, 19, 2457; Young, A. P. (1979). Phys. Rev. B, $19,1855$.

[25] Smith, G. S., Sirota, E. B., Safinya, C. R., Plano, R. J., \& Clark, N. A. (1990). J. Chem. Phys., 92, 4519.

[26] Pindak, R., Seul, M., \& Heinekamp, S. (unpublished).

[27] Chao, C. Y., Maclennan, J. E., Pang, J. Z., Hui, S. W., \& Ho, J. T. (1998). Phys. Rev. $E, 57,6757$.

[28] Chao, C. Y., Lo, C. R., \& Ho, J. T. (2000). Phys. Rev. E, 61, 5407. 
Copyright of Molecular Crystals \& Liquid Crystals is the property of Taylor \& Francis Ltd. The copyright in an individual article may be maintained by the author in certain cases. Content may not be copied or emailed to multiple sites or posted to a listserv without the copyright holder's express written permission. However, users may print, download, or email articles for individual use. 